\title{
The differentiation of hMSCs counteracts their migration capability and pro-angiogenic effects in vitro
}

\author{
A. SCHERZED, S. HACKENBERG, K. FROELICH, K. RAK, P. SCHENDZIELORZ, \\ T. GEHRKE, R. HAGEN and N. KLEINSASSER \\ Department of Otorhinolaryngology, Plastic, Aesthetic and Reconstructive Head and Neck Surgery, \\ Julius Maximilian University of Wuerzburg, D-97080 Wuerzburg, Germany \\ Received February 16, 2015; Accepted September 10, 2015
}

DOI: $10.3892 /$ or.2015.4383

\begin{abstract}
Human mesenchymal stem cells (hMSCs) have been applied therapeutically in numerous clinical trials. The pro-angiogenic effects of hMSCs, as well as their strong tumor tropism, have been shown both in vitro and in vivo. Some studies suggest using hMSCs as potential drug-carriers for tumor therapy. In previous investigations by our group, the pro-tumorigenic effects of hMSCs on head and neck squamous cell carcinoma (HNSCC) were shown. However, the influence of hMSCs on tumor vascularization as well as the possibility of its inhibition are yet to be elucidated. The cytokine patterns of the HNSCC cell line FaDu, native hMSCs (hMSCs-nat), hMSCs differentiated into adipocytes (hMSCs-adip) and osteocytes (hMSCs-ost) were evaluated. Human umbilical vein endothelial cells (HUVECs) were co-cultured with $\mathrm{FaDu}$ cells, hMSCs-nat, hMSCs-adip and hMSCs-ost. The capillary tube formation assay was applied. Furthermore, the migration capability of hMSCs-nat, hMSCs-adip and hMSCs-ost towards FaDu cells was measured in a Transwell system. Spheroids were cultured from hMSCs-nat, FaDu cells and DiI-labeled HUVECs. FaDu cells, hMSCs-nat, hMSCs-adip and hMSCs-ost released a wide range of cytokines and growth factors, e.g., IL-6, IL-8, IL-10, GRO and MCP. In the capillary tube formation assay, HUVECs generated significantly longer tubes after co-cultivation with hMSCs-nat as compared to HUVECs alone and FaDu. Differentiation into adipocytes and osteocytes counteracted the tube formation. The adipogenic differentiation did not alter hMSC motility, whereas osteogenic differentiation significantly inhibited hMSC migration. Generation of multi-cellular spheroids from hMSCs-nat, FaDu cells and DiI-labeled HUVECs was possible. Florescence
\end{abstract}

Correspondence to: Professor Norbert Kleinsasser, Department of Otorhinolaryngology, Plastic, Aesthetic and Reconstructive Head and Neck Surgery, Julius Maximilian University of Wuerzburg, 11 Josef-Schneider Street, D-97080 Wuerzburg, Germany

E-mail: kleinsasser_n@ukw.de

Key words: HNSCC, mesenchymal stem cells, angiogenesis, cytokines, human umbilical vein endothelial cells microscopy revealed that all HUVECs were present in the spheroid core. Taken together, hMSCs-nat have a pro-angiogenic effect. The effects are counteracted by the differentiation of hMSCs towards osteogenic and adipogenic lineages. The differentiation of stem cells into different lineages may be a promising solution to generating carriers for cancer therapy without pro-tumorigenic properties.

\section{Introduction}

Normal and pathological tissue growth depends on sufficient angiogenesis. Especially in cancer development, neoangiogenesis plays a key role. During cancer progression two different phases of cancer nutrition can be distinguished. At the beginning of cancer development, nutrition is supplied by diffusion. At a critical tumor volume of $1-2 \mathrm{~mm}^{3}$, diffusion is no longer sufficient (1). Further tumor progression requires a vascular network, which is induced by tumor cells via cytokines and growth factors. This phenomenon is called the 'angiogenic switch' (2). During tumor progression, the tissue behaves like an inflamed wound (3). Hence, the migration of several cell types, including human mesenchymal stem cells (hMSCs), is induced. The latter is even equipped with a pro-angiogenic capability. In a study conducted by Kinnaird et al, hMSCs released several cytokines responsible for angiogenesis, e.g., vascular endothelial growth factor (VEGF), placental growth factor (PIGF) and monocyte chemoattractant protein-1 (MCP-1) (4). Further cytokines were detected in hMSC-conditioned medium, especially interleukin 6 (IL-6) (5), which is a multifunctional cytokine responsible for immune response as well as hematopoiesis $(6,7)$.

hMSCs are self-renewing multipotent cells with the ability to differentiate into various mesenchymal cell types, e.g., osteoblasts, chondrocytes and adipocytes (8). Recent data indicate that hMSCs are pericytes whose pleiotropic nature allows them to sense and respond to inflammatory processes in their microenvironment (9). The migratory behavior of systemically administered hMSCs enables new therapeutic options, especially in the field of drug delivery. Myers et al found that hMSCs have been therapeutically applied in more than 200 active clinical trials worldwide (9). However, using hMSCs as a drug-carrier for cancer therapy is complicated, since data concerning cancer and hMSC interaction are contradictory. On the one hand, there are studies postulating tumor 
progression and enhancement of tumor metastatic potential by hMSCs via cell-cell contact as well as via secretion of cytokines and growth factors (10-12). On the other hand, reports on the anti- tumorigenic effects of hMSCs exist as well $(13,14)$. Different sources of stem cells with variable differentiation status may contribute to the divergent results. The aim of the present study was to investigate the cytokine patterns of the HNSCC cell line FaDu, native hMSCs (hMSCs-nat), hMSCs differentiated into adipocytes (hMSCs-adip) and osteocytes (hMSCs-ost). Furthermore, the migration capabilities as well as the angiogenic effects of hMSCs-nat, hMSCs-adip and hMSCs-ost were evaluated in a co-culture with human umbilical vein endothelial cells (HUVECs).

\section{Materials and methods}

hMSC isolation and culture. hMSCs were isolated from five voluntary patients undergoing surgery at the Department of Orthopedics, Koenig-Ludwig-Haus (Wuerzburg). Informed consent was provided by all patients. The study was approved by the Ethics Committee of the Medical Faculty of the University of Wuerzburg (12/06). Ficoll density gradient centrifugation (30 min, 1,300 rpm, density, 1,077 g/ml; Biochrom AG, Berlin, Germany) was performed as previously described (15). After centrifugation, cells in the interphase were collected. Several washing steps with phosphate-buffered saline (PBS) (Roche Diagnostics GmbH, Mannheim, Germany) containing $2 \%$ fetal calf serum (FCS) (Linaris, Wertheim-Bettingen, Germany) followed. The cells were resuspended in Dulbecco's modified Eagle's medium (DMEM) containing $10 \%$ FCS, $1 \%$ penicillin and streptomycin (Sigma-Aldrich, Schnelldorf, Germany). Flow cytometry (FACSCanto ${ }^{\mathrm{TM}}$; BD Bioscience, Heidelberg, Germany) was performed to show the characteristic surface markers of hMSCs. Furthermore, osteogenic and adipogenic differentiation was carried out as described previously (16). Osteogenic medium consisted of expansion medium (DMEM-EM) supplemented with $10^{-7} \mathrm{M}$ dexamethasone, $10^{-3} \mathrm{M} \beta$-glycerophosphate and 2-4 M ascorbate-2-phosphate (all from Sigma-Aldrich). Adipogenic differentiation medium was prepared by the addition of $10^{-7} \mathrm{M}$ dexamethasone and $10^{-9} \mathrm{~g} / \mathrm{ml}$ recombinant human insulin (all from Sigma-Aldrich) to DMEM-EM. Osteogenic differentiation was confirmed by Alizarin Red staining and adipogenic differentiation by Oil Red O staining.

HUVEC isolation and culture. HUVECs were isolated from human umbilical veins of three voluntary patients via $0.25 \%$ trypsin (Gibco Invitrogen, Karlsruhe, Germany) digestion. First, the umbilical vein was washed in D-Hank's solution. Next, a 10 -min perfusion with a $0.25 \%$ trypsin solution at $37^{\circ} \mathrm{C}$ followed. The enzyme reaction was blocked by addition of FCS. After a centrifugation step at $1,000 \mathrm{rpm}$ for $15 \mathrm{~min}$, the cells were cultured in endothelial cell growth medium with supplements (ECGM) (Provitro GmbH, Berlin, Germany) and $1 \%$ penicillin/streptomycin.

HNSCC cell line FaDu. The head and neck squamous carcinoma (HNSCC) cell line FaDu was used (17). The cultivation of the FaDu cells was performed in DMEM-EM at $37^{\circ} \mathrm{C}$ with $5 \% \mathrm{CO}_{2}$. Medium was replaced every other day.
Generation of multi-cellular spheroids.HUVECs were labeled with the fluorochrome 1,1'-dioctadecyl-3,3,3',3'-tetramethylindo-carbocyanine perchlorate (DiI) (Gibco Invitrogen). The generation of multi-cellular spheroids was performed as described previously (16). After solidification of $0.1 \%$ soft agar (Sigma-Aldrich) in a 96-multiwell plate, $0.2 \mathrm{ml}$ DMEM-EM containing $3 \times 10^{3} \mathrm{FaDu}$ cells and $3 \times 10^{3}$ HUVECs or $2 \times 10^{3}$ FaDu, $2 \times 10^{3}$ HUVECs and $2 \times 10^{3}$ hMSCs-nat were added.

Cytokine analysis of FaDu cells, hMSCs-nat, hMSCs-adip and hMSCs-ost with the dot blot assay. The dot blot assay (RayBiotec Inc., Norcross, GA, USA) is a semiquantitative method for investigating the cytokine secretion of cells. FaDu cells, hMSCs-nat, hMSCs-ost and hMSCs-adip were cultured in DMEM without supplements. Next, the supernatants were collected and analyzed for the presence of cytokines. The assay was performed according to the manufacturer's protocol. The observation of labeled cytokines was achieved via enhanced chemiluminescence using detection buffer and exposure to an X-ray film. The cytokines are represented as dots with different intensity and growth size.

hMSC migration towards FaDu cells. The migration assay was assessed in Transwells (Corning Incorporated Costar, Wiesbaden, Germany), which were coated with 50\% Matrigel (Sigma-Aldrich). hMSCs-nat, hMSCs-adip or hMSCs-ost were coated on the top surface of the membrane and incubated with DMEM without FCS for $24 \mathrm{~h}$ at $37^{\circ} \mathrm{C}$ with $5 \% \mathrm{CO}_{2}$. A total of $5 \times 10^{4} \mathrm{FaDu}$ cells was added to the bottom of the well plate, followed by another incubation period for $24 \mathrm{~h}$. A cotton swab was used to remove the non-migratory cells on the upper membrane. The migrated cells, which collected on the lower surface of the membrane, were stained with $1 \%$ crystal violet (Sigma-Aldrich) for $25 \mathrm{~min}$. Another washing step with aqua destillata followed. The cells were detached by incubation with $500 \mu 110 \%$ acetic acid for $20 \mathrm{~min}$. Extinction was measured at $570 \mathrm{~nm}$ using a multi-plate reader (Titertek Multiskan Plus MKII; Labsystems, Helsinki, Finland). The migration of hMSCs towards DMEM-FCS served as the control. The results of the control group were defined as $100 \%$.

Capillary tube formation assay. hMSCs-nat, hMSCs-adip and hMSCs-ost were incubated on the bottom of $\mu$-Slide Angiogenesis (Ibidi, Munich, Germany) for $2 \mathrm{~h}$. Next, $50 \%$ Matrigel (Sigma-Aldrich) was added to the top of each cell system. After $30 \mathrm{~min}, 3 \times 10^{3}$ DiI-labeled HUVECs were coated on the Matrigel with ECGM. After $12 \mathrm{~h}$, the tube formation was evaluated by inverted microscopy (Leica DMI 4000B inverted microscope; Leica Microsystems, Wetzlar, Germany). The tube length was measured using ImageJ software (http://imagej.nih.gov/ij/).

Cultivation of hMSCs-nat on Matrigel. A total of 50\% Matrigel was added to the bottom of 48 -well plates for $30 \mathrm{~min}$. After solidification, $10^{4} \mathrm{hMSCs}$-nat were added and cultured with DMEM-EM. After $72 \mathrm{~h}$, the cells were observed by inverted microscopy.

Statistical analysis. All data were transferred to standard spreadsheets. Differences between groups were examined 

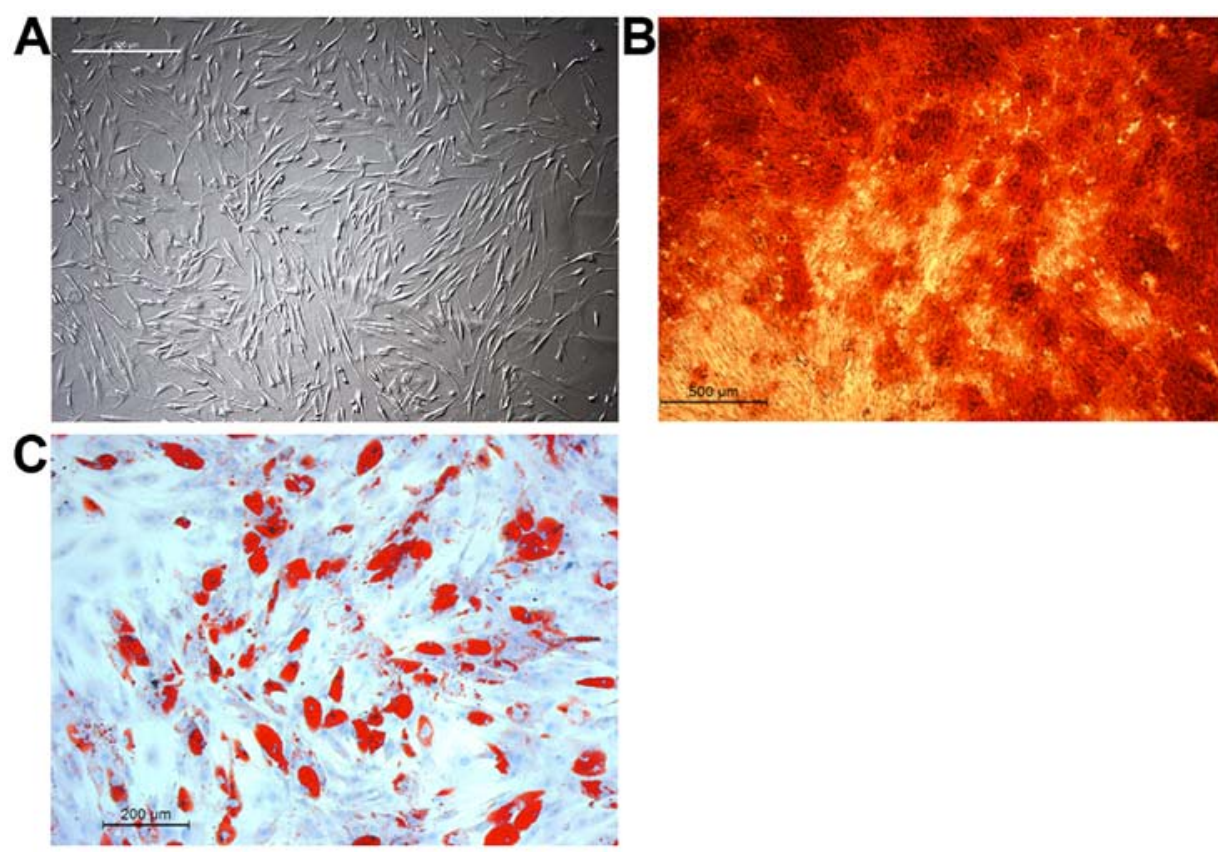

D

hMSC cell surface markers
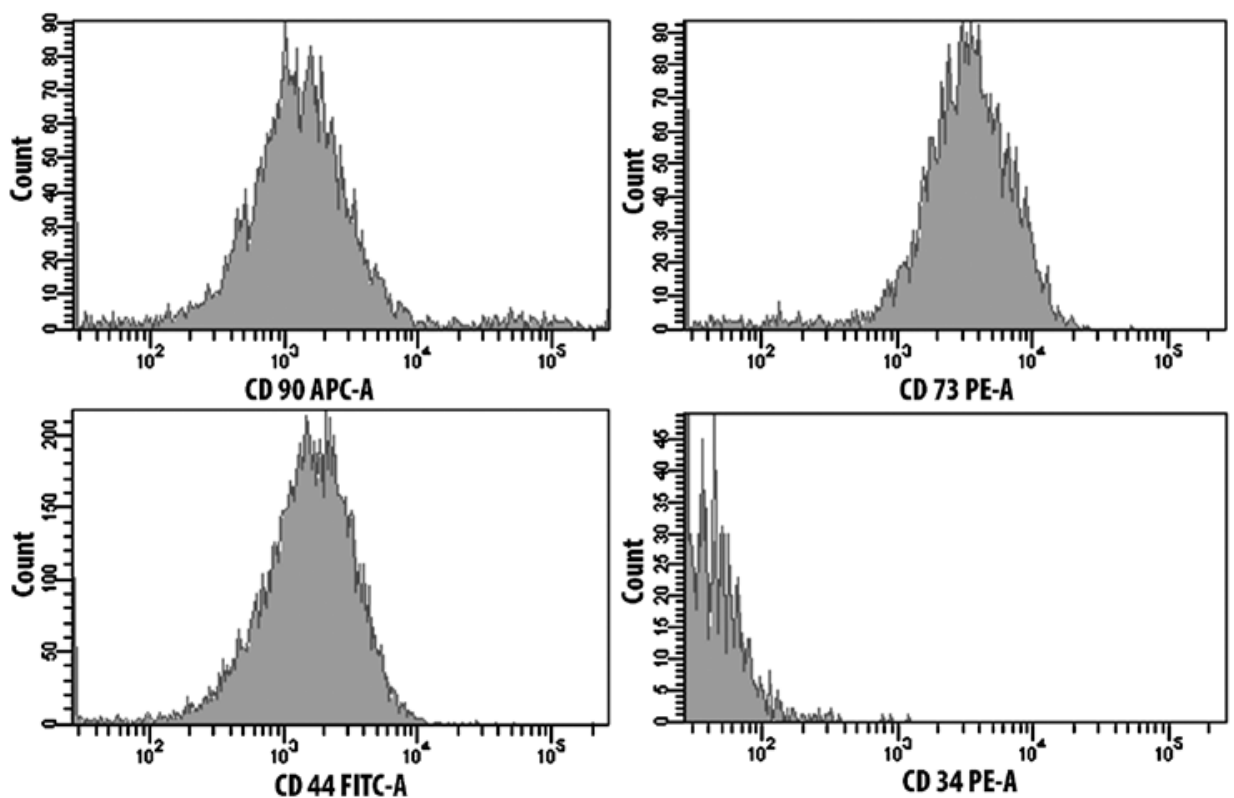

Figure 1. (A) Spindle-shaped hMSCs cultured in DMEM-EM. (B) hMSCs cultivated in osteogenic medium. The Alizarin Red staining was carried out to demonstrate the success of osteogenic differentiation. (C) hMSCs cultivated in adipogenic medium. The intracellular lipids were visualized by Oil Red O staining. (D) Cell surface markers were investigated by flow cytometric analysis. hMSCs were positive for CD90, CD73 and CD44 and negative or weakly positive for CD34.

for significance by the Kruskal-Wallis test using GraphPad Prism 6.0 statistics software (GraphPad Software, Inc., San Diego, CA, USA). P $<0.05$ was considered to indicate a statistically significant difference.

\section{Results}

hMSC morphology and differentiation. The hMSCs showed typical spindle-shaped structures. Cells were positive for CD105, CD90 and CD44, and negative for CD34. The differentiation into adipocytes was confirmed by Oil Red O staining with typical intracellular lipid vacuoles. The osteogenic differentiation was shown with Alizarin Red staining. The extracellular calcium deposits were stained red (Fig. 1).

Morphology of the spheroids. There was no difference in spheroid growth from the FaDu and HUVECs as compared to the spheroids from the FaDu cells, hMSCs-nat and HUVECs. Spheroids had a well-shaped structure. The mean diameter of the spheroids from the FaDu and the HUVECs was $383.4 \mu \mathrm{m}$. The mean diameter of the spheroids from the FaDu, hMSCs-nat and HUVECs was $405.6 \mu \mathrm{m}$. The differences between both groups were not statistically significant. Fluorescence microscopy revealed that almost all HUVECs 
Table I. Array map.

\begin{tabular}{cccclllllll}
\hline+ & + & - & - & ENA-78 & GCSF & GM-CSF & GRO & GRO- $\alpha$ & I-309 & IL-1 $\alpha$ IL-1 $\beta$ \\
+ & + & - & - & ENA-78 & GCSF & GM-CSF & GRO & GRO- $\alpha$ & I-309 & IL-1 $\alpha$ IL-1 $\beta$ \\
IL-2 & IL-3 & IL-4 & IL-5 & IL-6 & IL-7 & IL-8 & IL-10 & IL-12 & IL-13 & IL-15 IFN- $\gamma$ \\
IL-2 & IL-3 & IL-4 & IL-5 & IL-6 & IL-7 & IL-8 & IL-10 & IL-12 & IL-13 & IL-15 IFN- $\gamma$ \\
MCP-1 & MCP-2 & MCP-3 & MCSF & MCD & MIG & MIP-1d & RANTES SCF & SDF-1TA RC & TGF- $\beta 1$ \\
MCP-1 & MCP-2 & MCP-3 & MCSF & MCD & MIG & MIP-1d & RANTES SCF & SDF-1TA RC & TGF- $\beta 1$ \\
TNF- $\alpha$ & TNF- $\beta$ & EGF & IGF-1 & Angogenin Oncostatin M Thrombopoietin VEGF & PDGF-BB & Leptin & - & + \\
TNF- $\alpha$ & TNF- $\beta$ & EGF & IGF-1 & Angogenin & Oncostatin M & Thrombopoietin & VEGF & PDGF-BB & Leptin & - \\
\hline
\end{tabular}

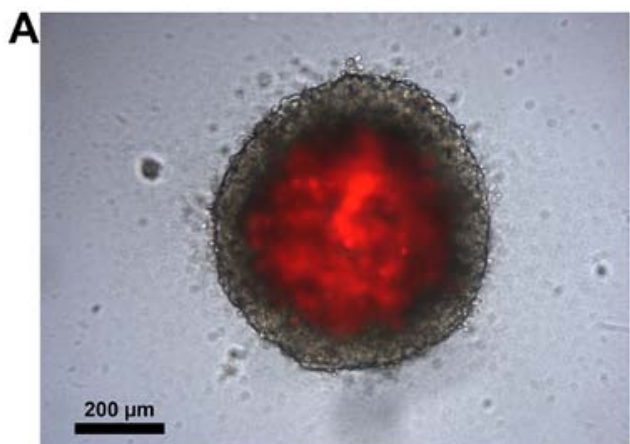

B
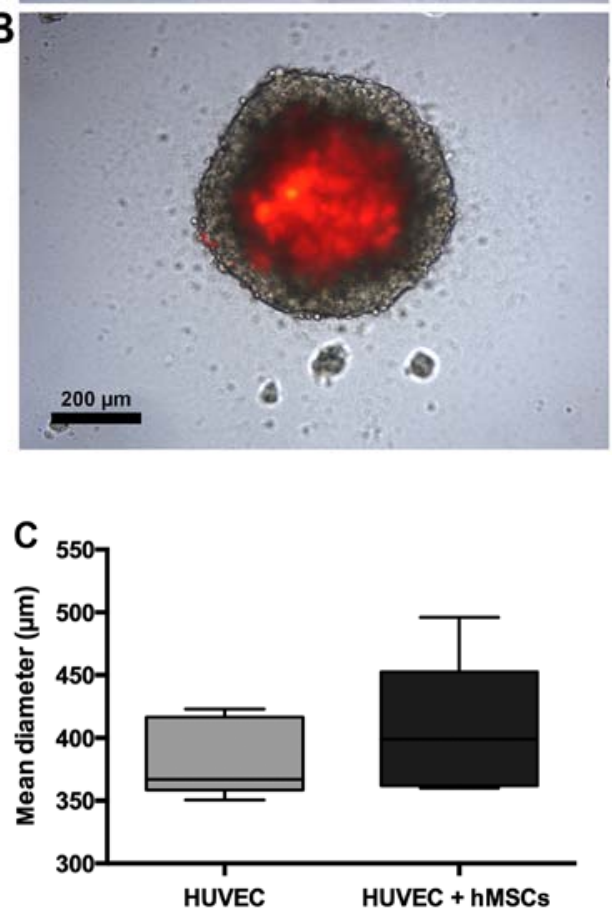

Figure 2. (A) Spheroids generated from FaDu cells and DiI-labeled HUVECs (B) Spheroids generated from FaDu cells, hMSCs-nat and DiI-labeled HUVECs. The labeled HUVECs were visualized by fluorescence microscopy. Almost all HUVECs were located in the center of the spheroids. (C) In both groups, the diameter of the spheroids was measured with Image J software. The diameters in each group did not differ significantly.

were located in the core of the spheroids. There were only very few HUVECs observed in the circumference (Fig. 2).

Cytokine analysis of hMSCs-nat, hMSCs-adip, hMSCs-ost and FaDu cells. FaDu cells, hMSCs-nat, hMSCs-adip and hMSCs-ost released different cytokines and growth factors responsible for inflammation, angiogenesis and chemo- taxis (Table I). Dots of the following cytokines had a strong intensity in the FaDu cells: interleukin (IL)-3, IL-8, IL-10, growth regulated oncogene (GRO)- $\alpha$, RANTES, macrophage colony-stimulating factor (MCSF), oncostatin $\mathrm{M}$ and tumor necrosis factor (TNF)- $\alpha$.

Dots of the following cytokines had a strong intensity in hMSCs-nat: IL-3, IL-6, IL-8, IL-10, GRO, GRO- $\alpha$, monocyte chemotactic protein (MCP)-1, MCSF, RANTES, TNF- $\alpha$ and oncostatin $\mathrm{M}$.

Dots of the following cytokines had a strong intensity in the hMSCs-adip: IL-3, IL-6, IL-8, IL-10, GRO, GRO- $\alpha$, MCP-1, MCSF, RANTES, TNF- $\alpha$, TNF- $\beta$, oncostatin M, platelet-derived growth factor (PDGF)-BB and leptin.

Dots of the following cytokines had a strong intensity in the hMSCs-ost: IL-3, IL-6, IL-8, C-X-C motif chemokine 5, GRO, GRO- $\alpha$, MCP-1, MCSF, RANTES, TNF- $\alpha$, TNF- $\beta$, angiogenin, oncostatin M, PDGF-BB and leptin (Fig. 3).

Migration of hMSCs-nat, hMSCs-adip and hMSCs-ost towards FaDu cells. The migration assay was conducted in order to evaluate the differences between hMSCs-nat, hMSCs-adip and hMSCs-ost. The results of the control group (migration towards DMEM + 10\% FCS) were defined as $100 \%$. hMSCs-nat showed a significant enhancement in migration by $52 \%$ towards the FaDu cells. The differentiation of hMSCs into adipocytes (hMSCs-adip) did not counteract the migration enhancement as noted in the hMSC-nat.

The differentiation of hMSCs into osteocytes (hMSCs-ost) attenuated the migration capability significantly as compared to the migration of hMSC-nat and hMSC-adip. However, there were no significant differences between hMSCs-ost and the control group (Fig. 4).

Capillary tube formation assay on Matrigel. HUVECs formed tube-like structures on the Matrigel, and the lengths of these tubes were measured with ImageJ software. In co-culture with hMSCs-nat, HUVECs developed significantly longer tube-like structures as compared to the HUVECs alone or co-cultured with the FaDu cells. The formation of tube-like structures was counteracted by the co-cultivation with hMSCs-adip and hMSCs-ost. The tube length was significantly attenuated after the co-cultivation of the HUVECs with hMSCs-adip or hMSCs-ost. The lengths as well as the ability to build tube-like structures were negatively affected (Fig. 5).

Cultivation of hMSCs-nat on Matrigel. After the cultivation of hMSCs-nat on Matrigel, some of the cells took the shape of 

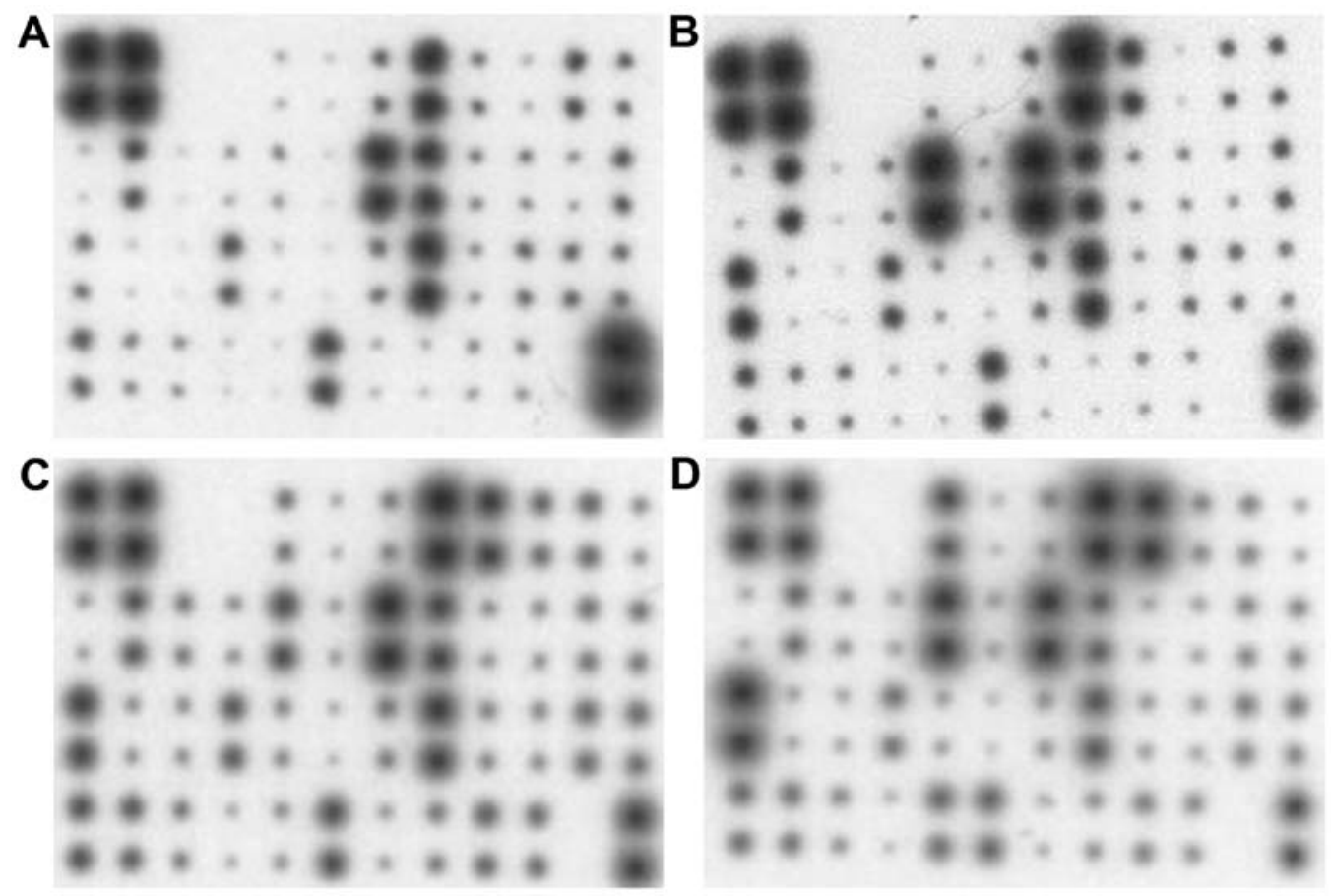

Figure 3. Dot blot assay of (A) FaDu cells, (B) hMSCs-nat, (C) hMSCs-adip and (D) hMSCs-ost cultivated with DMEM without supplements. After a period of $24 \mathrm{~h}$, the cytokine release was investigated. In order to identify the different dots, a table included in the manufacturer's protocol was used (Table I). hMSCs-nat, FaDu cells, hMSCs-adip and hMSCs-ost released several cytokines.

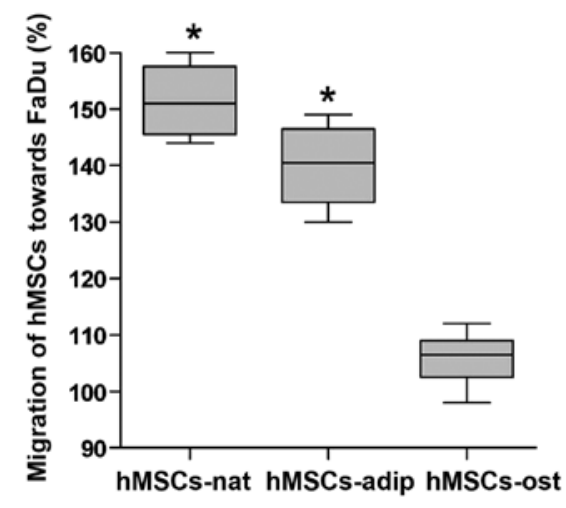

Figure 4. A Transwell system was used to evaluate the migration of hMSCs-nat, hMSCs-adip and hMSCs-ost towards FaDu cells. The results of the control group (migration towards DMEM + 10\% FCS) were defined as $100 \%$. The migration of hMSCs-nat and hMSCs-adip was enhanced significantly as compared to the control and hMSCs-ost. The osteogenic differentiation counteracted hMSC migration towards the FaDu cells. This was $52 \%$ more than the migration towards DMEM-EM. The differentiation of hMSCs into osteocytes significantly counteracted the migration capability.

a spheroid. From these spheroids tube-like structures sprouted out. The length of the tube-like structures was not significantly higher as compared to the HUVECs (Fig. 6).

\section{Discussion}

The induction of angiogenesis is one of the six hallmarks of cancer as proposed by Hanahan and Weinberg. It differs significantly in tumors compared to normal physiological processes. In cases such as wound healing or female reproductive cycling the angiogenesis process is turned on transiently, whereas in cancer progression the so-called 'angiogenic switch' is acti- vated permanently (18). In addition to nutrition and oxygen supply, neovascularization is a prerequisite for cancer cells to circulate and induce metastasis (19). Furthermore, tumor vasculature may be a reason for cancer therapy failure. The vessels can be leaky and arranged chaotically (20). The cancer vasculature system is abnormal with heterogeneous perfusion areas, with the poorly perfused regions not being reached by anticancer drugs (21). This may lead to cancer resistance towards anticancer drugs. In the process of angiogenesis, interactions between tumor cells with the non-malignant tumor stroma are fundamental. Cytokines and growth factors such as VEGF, fibroblast growth factors (FGF), angiopoietin (Ang)-1 and Ang2, IL-6 and IL-8 are all part of the orchestration (22). The dot blot assay revealed the presence of these proteins in the present study. hMSCs as well as FaDu cells were able to release cytokines and growth factors. Cancer and stroma interact by direct cell-cell contact as well as by soluble factors, which implies their symbiotic relationship. In tissue engineering, especially in cardiology and the regeneration of cardiomyocytes, hMSCs have already demonstrated their pro-angiogenic features. These effects were mediated via secretion of cytokines such as VEGF, angiopoietin, SDF-1 or integrin-linked kinase (23-25). In ischemia studies, hMSCs showed their pro-angiogenic effect, which was resolved via expression of hypoxia inducible factor-1 $\alpha(26,27)$. The cultivation of hMSCs on Matrigel revealed capillary-like formation. The potential of hMSCs to build capillary networks was shown by several other groups (28-30). The generation of this capillary network was induced in the presence of VEGF and insulin-like growth factors. These results demonstrate the direct involvement of hMSCs in angiogenesis by forming capillary-like structures.

Cancer treatment must target several aspects of the tumor including the tumor microenvironment. Here, anti-angiogenic 

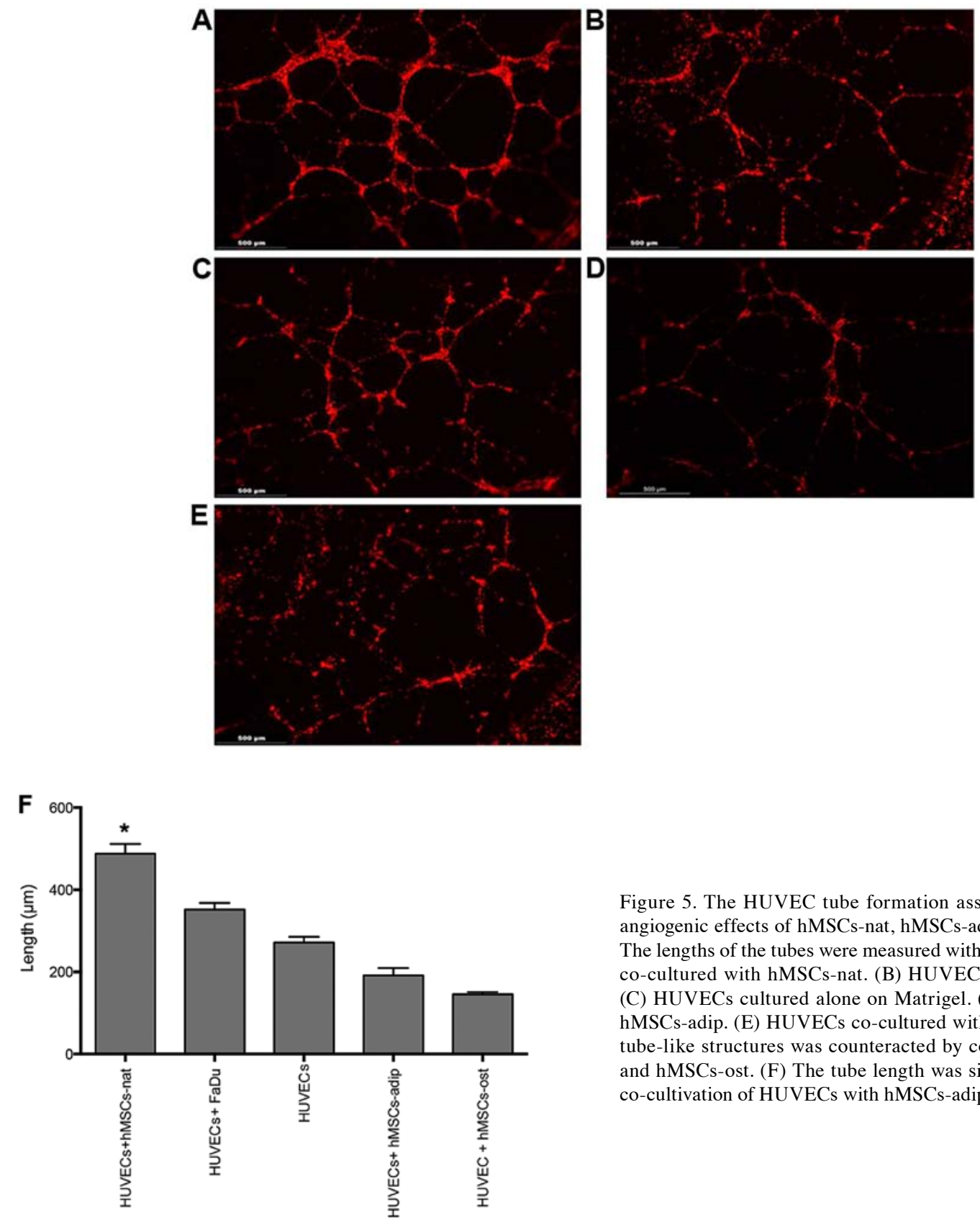

Figure 5. The HUVEC tube formation assay was used to investigate the angiogenic effects of hMSCs-nat, hMSCs-adip, hMSCs-ost and FaDu cells. The lengths of the tubes were measured with ImageJ software. (A) HUVECs co-cultured with hMSCs-nat. (B) HUVECs co-cultured with FaDu cells. (C) HUVECs cultured alone on Matrigel. (D) HUVECs co-cultured with hMSCs-adip. (E) HUVECs co-cultured with hMSCs-ost. The formation of tube-like structures was counteracted by co-cultivation with hMSCs-adip and hMSCs-ost. (F) The tube length was significantly attenuated after the co-cultivation of HUVECs with hMSCs-adip or hMSCs-ost.
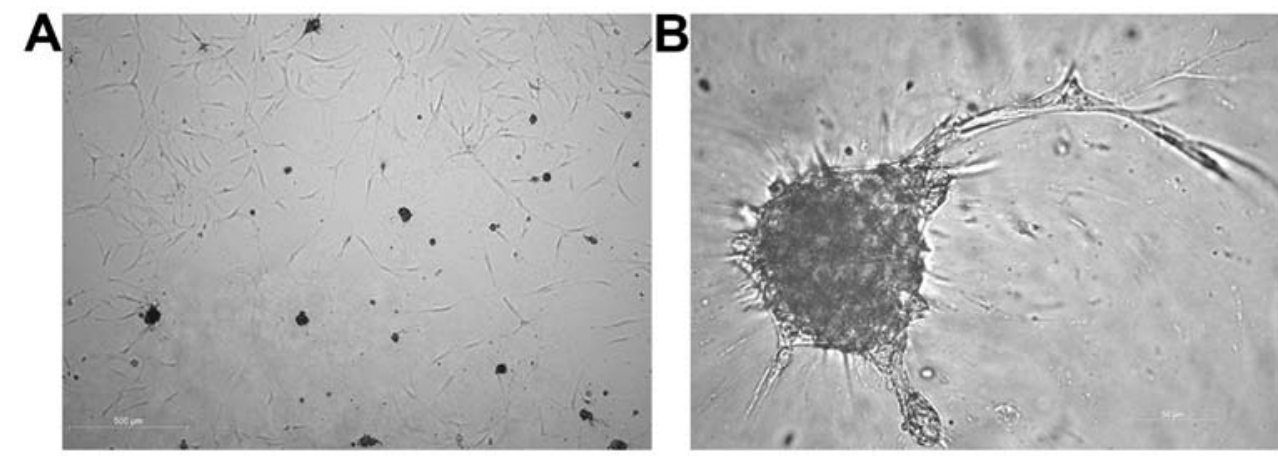

Figure 6. hMSCs cultured on Matrigel. (A) The hMSCs-nat showed 2-dimensional as well as 3-dimensional growth behavior. (B) Higher magnification of a 3-dimensional spheroid. From these spheroids tube-like structures sprouted out.

medications have recently gained popularity for the treatment of several tumor entities (31-33). However, targeted tumor therapy requires an appropriate vehicle, and hMSCs show strong tumor tropism. Thus, hMSCs are often considered to be 
an adequate candidate for tumor therapy (34-36). Yet, the data in the literature concerning the applicability of hMSCs are divergent. The majority of the studies show a pro-tumorigenic effect of hMSCs. These effects are induced in various different ways. Zhang et al demonstrated an induction of autophagy and thereby an enhancement of cancer cell survival (37). Jung et al demonstrated an induction of epithelial-mesenchymal transition by hMSCs, with a consecutive enhancement of the metastatic potential of cancer cells (38). Cancer growth was shown to be promoted by cytokine secretion from hMSCs such as oncostatin M secretion (39). Cytokines appear to be primarily responsible for cancer progression and metastasis.

In the minority of studies, cancer inhibition by hMSCs was described. In a study conducted by Chen et al, hMSCs derived from placenta were transfected with recombinant adenoviruses expressing pigment epithelium-derived factor (PEDF), and these hMSCs were able to inhibit melanoma cell growth. This anticancer activity was discussed to be the result of PEDF expression (40). Li et al demonstrated inhibition of gastric cancer cells by hMSCs derived from human foreskin (41).

The divergent results in the current literature may be due to the use of hMSCs from different sources as well as the donor's age. Nevertheless, hMSCs as a vehicle for cancer therapy have been used in various studies (42-44), particularly as an engineered vehicle administered via viral transfection. In a study conducted by Martinez-Quintanilla et al engineered hMSCs were able to co-express the pro-drug converting enzyme as well as the herpes simplex virus thymidine kinase (HSV-TK) and a potent and secretable variant of tumor necrosis factor apoptosis-inducing ligand (S-TRAIL). Via these capabilities caspase-mediated cancer cell death was inducted (44). Other studies used retroviral transfection of hMSCs with promising results $(45,46)$. However, such viral vectors may have risk factors, e.g., integration into the host chromosomes. Thus non-viral transfected hMSCs or native hMSCs with antitumor properties may be the perfect candidates for tumor-targeted therapy.

The present study revealed a pro-angiogenic effect of hMSCs-nat. Interestingly, the differentiation of hMSCs counteracted the pro-angiogenic effects as well as their migration capability. The cause of this phenomenon needs to be investigated to ascertain an alteration of the hMSC cytokine pattern during differentiation. Counteracting the pro-angiogenic effects of hMSCs may be suitable for the generation of a 'safe vehicle' for future targeted tumor therapy.

\section{Acknowledgements}

The present study was supported by Rudolf Bartling Stiftung, the Department of Orthopaedic Surgery, Koenig-Ludwig-Haus (Professor Maximilian Rudert, Director) and Dr Marianne Schmidt, Department of Otorhinolaryngology, Plastic, Aesthetic and Reconstructive Head and Neck Surgery, Julius Maximilian University of Wuerzburg, Germany.

\section{References}

1. Folkman $\mathrm{J}$ : What is the evidence that tumors are angiogenesis dependent? J Natl Cancer Inst 82: 4-6, 1990.

2. Bergers $G$ and Benjamin LE: Tumorigenesis and the angiogenic switch. Nat Rev Cancer 3: 401-410, 2003.
3. Dvorak HF: Tumors: Wounds that do not heal. Similarities between tumor stroma generation and wound healing. N Engl J Med 315: 1650-1659, 1986.

4. Kinnaird T, Stabile E, Burnett MS, Shou M, Lee CW, Barr S, Fuchs S and Epstein SE: Local delivery of marrow-derived stromal cells augments collateral perfusion through paracrine mechanisms. Circulation 109: 1543-1549, 2004.

5. Hung SC, Pochampally RR, Chen SC, Hsu SC and Prockop DJ: Angiogenic effects of human multipotent stromal cell conditioned medium activate the PI3K-Akt pathway in hypoxic endothelial cells to inhibit apoptosis, increase survival, and stimulate angiogenesis. Stem Cells 25: 2363-2370, 2007.

6. Kanazawa T, Nishino H, Hasegawa M, Ohta Y, Iino Y, Ichimura K and Noda Y: Interleukin-6 directly influences proliferation and invasion potential of head and neck cancer cells. Eur Arch Otorhinolaryngol 264: 815-821, 2007.

7. Kishimoto T: The biology of interleukin-6. Blood 74: 1-10, 1989.

8. Caplan AI: Mesenchymal stem cells. J Orthop Res 9: 641-650, 1991.

9. Myers JT, Barkauskas DS and Huang AY: Dynamic imaging of marrow-resident granulocytes interacting with human mesenchymal stem cells upon systemic lipopolysaccharide challenge. Stem Cells Int 2013: 656839, 2013

10. Karnoub AE, Dash AB, Vo AP, Sullivan A, Brooks MW, Bell GW, Richardson AL, Polyak K, Tubo R and Weinberg RA: Mesenchymal stem cells within tumour stroma promote breast cancer metastasis. Nature 449: 557-563, 2007.

11. Lin WR, Brittan M and Alison MR: The role of bone marrow-derived cells in fibrosis. Cells Tissues Organs 188: $178-188,2008$

12. Rhodes LV, Muir SE, Elliott S, Guillot LM, Antoon JW, Penfornis P, Tilghman SL, Salvo VA, Fonseca JP, Lacey MR, et al: Adult human mesenchymal stem cells enhance breast tumorigenesis and promote hormone independence. Breast Cancer Res Treat 121: 293-300, 2010

13. Khakoo AY, Pati S, Anderson SA, Reid W, Elshal MF, Rovira II, Nguyen AT, Malide D, Combs CA, Hall G, et al: Human mesenchymal stem cells exert potent antitumorigenic effects in a model of Kaposi's sarcoma. J Exp Med 203: 1235-1247, 2006.

14. Zhu Y, Sun Z, Han Q, Liao L, Wang J, Bian C, Li J, Yan X, Liu Y, Shao C, et al: Human mesenchymal stem cells inhibit cancer cell proliferation by secreting DKK-1. Leukemia 23: 925-933, 2009.

15. Scherzed A, Hackenberg S, Froelich K, Rak K, Technau A, Radeloff A, Nöth U, Koehler C, Hagen R and Kleinsasser N: Effects of salinomycin on human bone marrow-derived mesenchymal stem cells in vitro. Toxicol Lett 218: 207-214, 2013.

16. Scherzed A, Hackenberg S, Radeloff A, Froelich K, Rak K, Hagen R and Kleinsasser N: Human mesenchymal stem cells promote cancer motility and cytokine secretion in vitro. Cells Tissues Organs 198: 327-337, 2013.

17. Rangan SR: A new human cell line $(\mathrm{FaDu})$ from a hypopharyngeal carcinoma. Cancer 29: 117-121, 1972

18. Hanahan D and Weinberg RA: Hallmarks of cancer: The next generation. Cell 144: 646-674, 2011.

19. Zetter BR: Angiogenesis and tumor metastasis. Annu Rev Med 49: 407-424, 1998

20. Grantab RH and Tannock IF: Penetration of anticancer drugs through tumour tissue as a function of cellular packing density and interstitial fluid pressure and its modification by bortezomib. BMC Cancer 12: 214, 2012

21. Jain RK: Normalizing tumor microenvironment to treat cancer: Bench to bedside to biomarkers. J Clin Oncol 31: 2205-2218, 2013.

22. Gupta MK and Qin RY: Mechanism and its regulation of tumor-induced angiogenesis. World J Gastroenterol 9: 1144-1155, 2003.

23. Copland IB: Mesenchymal stromal cells for cardiovascular disease. J Cardiovasc Dis Res 2: 3-13, 2011.

24. Zhang D, Fan GC, Zhou X, Zhao T, Pasha Z, Xu M, Zhu Y, Ashraf $M$ and Wang Y: Over-expression of CXCR4 on mesenchymal stem cells augments myoangiogenesis in the infarcted myocardium. J Mol Cell Cardiol 44: 281-292, 2008.

25. Wen Z, Huang W, Feng Y, Cai W, Wang Y, Wang X, Liang J, Wani M, Chen J, Zhu P, et al: MicroRNA-377 regulates mesenchymal stem cell-induced angiogenesis in ischemic hearts by targeting VEGF. PLoS One 9: e104666, 2014.

26. Hua J, He ZG, Qian DH, Lin SP, Gong J, Meng HB, Yang TS, Sun W, Xu B, Zhou B, et al: Angiopoietin-1 gene-modified human mesenchymal stem cells promote angiogenesis and reduce acute pancreatitis in rats. Int J Clin Exp Pathol 7: 3580-3595, 2014. 
27. Park IS, Chung PS and Ahn JC: Enhanced angiogenic effect of adipose-derived stromal cell spheroid with low-level light therapy in hind limb ischemia mice. Biomaterials 35: 9280-9289, 2014

28. Jazayeri M, Allameh A, Soleimani M, Jazayeri SH, Piryaei A and Kazemnejad S: Molecular and ultrastructural characterization of endothelial cells differentiated from human bone marrow mesenchymal stem cells. Cell Biol Int 32: 1183-1192, 2008.

29. Aguirre A, Planell JA and Engel E: Dynamics of bone marrow-derived endothelial progenitor cell/mesenchymal stem cell interaction in co-culture and its implications in angiogenesis Biochem Biophys Res Commun 400: 284-291, 2010.

30. Malinowski M, Pietraszek K, Perreau C, Boguslawski M, Decot V, Stoltz JF, Vallar L, Niewiarowska J, Cierniewski C, Maquart FX, et al: Effect of lumican on the migration of human mesenchymal stem cells and endothelial progenitor cells: Involvement of matrix metalloproteinase-14. PLoS One 7: e50709, 2012.

31. García-Alfonso P, Grande E, Polo E, Afonso R, Reina JJ, Jorge M, Campos JM, Martínez V, Angeles C and Montagut C: The role of antiangiogenic agents in the treatment of patients with advanced colorectal cancer according to K-RAS status. Angiogenesis 17: 805-821, 2014.

32. Wang Z, Wang M, Yang F, Nie W, Chen F, Xu J and Guan X: Multitargeted antiangiogenic tyrosine kinase inhibitors combined to chemotherapy in metastatic breast cancer: A systematic review and meta-analysis. Eur J Clin Pharmacol 70: 531-538, 2014.

33. Ohara T, Noma K, Urano S, Watanabe S, Nishitani S, Tomono Y Kimura F, Kagawa S, Shirakawa Y and Fujiwara T: A novel synergistic effect of iron depletion on antiangiogenic cancer therapy. Int J Cancer 132: 2705-2713, 2013.

34. Xin H, Kanehira M, Mizuguchi H, Hayakawa T, Kikuchi T, Nukiwa $\mathrm{T}$ and Saijo Y: Targeted delivery of CX3CL1 to multiple lung tumors by mesenchymal stem cells. Stem Cells 25 $1618-1626,2007$.

35. Kolluri KK, Laurent GJ and Janes SM: Mesenchymal stem cells as vectors for lung cancer therapy. Respiration 85: 443-451, 2013

36. Kanehira M, Xin H, Hoshino K, Maemondo M, Mizuguchi H, Hayakawa T, Matsumoto K, Nakamura T, Nukiwa T and Saijo Y: Targeted delivery of NK4 to multiple lung tumors by bone marrow-derived mesenchymal stem cells. Cancer Gene Ther 14 894-903, 2007.
37. Zhang MH, Hu YD, Xu Y, Xiao Y, Luo Y, Song ZC and Zhou J: Human mesenchymal stem cells enhance autophagy of lung carcinoma cells against apoptosis during serum deprivation. Int J Oncol 42: 1390-1398, 2013.

38. Jung Y, Kim JK, Shiozawa Y, Wang J, Mishra A, Joseph J, Berry JE, McGee S, Lee E, Sun H, et al: Recruitment of mesenchymal stem cells into prostate tumours promotes metastasis. Nat Commun 4: 1795, 2013.

39. Lee MJ, Heo SC, Shin SH, Kwon YW, Do EK, Suh DS, Yoon MS and Kim JH: Oncostatin M promotes mesenchymal stem cell-stimulated tumor growth through a paracrine mechanism involving periostin and TGFBI. Int J Biochem Cell Biol 45: 1869-1877, 2013

40. Chen Q, Cheng P, Song N, Yin T, He H, Yang L, Chen X and Wei Y: Antitumor activity of placenta-derived mesenchymal stem cells producing pigment epithelium-derived factor in a mouse melanoma model. Oncol Lett 4: 413-418, 2012.

41. Li Y, Zhao Y, Cheng Z, Zhan J, Sun X, Qian H, Zhu W and Xu W: Mesenchymal stem cell-like cells from children foreskin inhibit the growth of SGC-7901 gastric cancer cells. Exp Mol Pathol 94: 430-437, 2013.

42. Katsuda T, Kosaka N, Takeshita F and Ochiya T: The therapeutic potential of mesenchymal stem cell-derived extracellular vesicles. Proteomics 13: 1637-1653, 2013.

43. Mader EK, Butler G, Dowdy SC, Mariani A, Knutson KL, Federspiel MJ, Russell SJ, Galanis E, Dietz AB and Peng KW: Optimizing patient derived mesenchymal stem cells as virus carriers for a phase I clinical trial in ovarian cancer. J Transl Med 11: 20, 2013.

44. Martinez-Quintanilla J, Bhere D, Heidari P, He D, Mahmood U and Shah K: Therapeutic efficacy and fate of bimodal engineered stem cells in malignant brain tumors. Stem Cells 31: 1706-1714, 2013.

45. Cavarretta IT, Altanerova V, Matuskova M, Kucerova L, Culig Z and Altaner C: Adipose tissue-derived mesenchymal stem cells expressing prodrug-converting enzyme inhibit human prostate tumor growth. Mol Ther 18: 223-231, 2010.

46. Kucerova L, Altanerova V, Matuskova M, Tyciakova S and Altaner C: Adipose tissue-derived human mesenchymal stem cells mediated prodrug cancer gene therapy. Cancer Res 67: 6304-6313, 2007. 